\title{
Social cognition in Williams syndrome: relations between performance on the social attribution task and cognitive and behavioral characteristics
}

\author{
Faye van der Fluit ${ }^{1}$, Michael S. Gaffrey ${ }^{2}$ and Bonita P. Klein-Tasman ${ }^{1 *}$ \\ Child Neurodevelopment Research Lab, Department of Psychology, University of Wisconsin-Milwaukee, Milwaukee, WI, USA \\ 2 Early Emotional Development Program, Department of Psychiatry, Washington University in St. Louis, St. Louis, MO, USA
}

\section{Edited by:}

Daniela Plesa Skwerer, Boston

University, USA

\section{Reviewed by:}

Ruth Ford, Griffith University, Australia

Ovsanna Leyfer, Boston University,

USA

\section{*Correspondence:}

Bonita P. Klein-Tasman, Department of Psychology, University of Wisconsin-Milwaukee, PO Box 413, Milwaukee, WI 53201, USA e-mail: bklein@uwm.edu
Williams syndrome (WS) is a developmental disorder of genetic origin, with characteristic cognitive and personality profiles. Studies of WS point to an outgoing and gregarious personality style, often contrasted with autism spectrum disorders; however, recent research has uncovered underlying social reciprocity difficulties in people with WS. Social information processing difficulties that underlie these social reciprocity difficulties have been sparsely examined. Participants in the current study included 24 children with WS ages 8 through 15. A lab-based measure of social perception and social cognition was administered (Social Attribution Test), as well as an intellectual functioning measure (KBIT-II) and parent reports of communication and reciprocal social skills (Social Communication Questionnaire, Social Responsiveness Scale). Relations between social cognition, cognitive abilities, and socialcommunication were examined. Results demonstrated relations between parent-reported social reciprocity and the typicality of the responses provided in the lab-based measure, even once variability in intellectual functioning was taken into account. Specifically, those individuals who produced narratives in response to the social attribution task (SAT) that were more similar to those described in previous studies of typically developing individuals were also reported to have fewer social reciprocity difficulties in the real world setting as reported by parents. In addition, a significant improvement in performance on the SAT was seen with added scaffolding, particularly for participants with stronger intellectual functioning. These findings indicate that difficulties interpreting the social dynamics between others in ambiguous situations may contribute to the social relationship difficulties observed in people with WS, above and beyond the role of intellectual functioning. Exploratory analyses indicated that performance by individuals with stronger intellectual functioning is improved with additional structure to a greater degree than for those with weaker intellectual functioning. Interventions that specifically target these social information processing of individuals with WS would likely be beneficial.

Keywords: Williams syndrome, social cognition, social reciprocity, behavioral phenotype, social attribution task

\section{INTRODUCTION}

Williams syndrome (WS) is a neurodevelopmental disorder of genetic origin, specifically resulting from the deletion of approximately 25 genes on chromosome 7q11.23 (Ewart et al., 1993; Hillier et al., 2003). Individuals with WS typically display a distinctive cognitive and personality profile. In terms of a cognitive profile, results of numerous studies have pointed to some degree of developmental delay in the majority of patients (Udwin and Yule, 1991; Greer et al., 1997; Mervis et al., 2000), with a pattern of relative strengths and weaknesses, including relatively stronger language, after a period of early delays, than would be expected given developmental level (Mervis and Bertrand, 1997; Mervis and Robinson, 2000) and a marked difficulty with visuospatial construction tasks (MacDonald and Roy, 1988; Wang et al., 1995; Mervis et al., 1999). The personality profile is characterized by high sociability and friendliness, as well as high levels of empathy (Dilts et al., 1990; Tomc et al., 1990; Gosch and Pankau, 1997; Klein-Tasman and
Mervis, 2003). Particular genes have been identified as being influential in the development of certain physical and behavioral traits commonly seen in WS, including connective tissue and cardiovascular abnormalities (Ewart et al., 1993), distinctive craniofacial features (Osborne et al., 1999; Tassabehji et al., 2005), difficulties in visuospatial abilities (Frangiskakis et al., 1996), and lower cognitive abilities (Morris et al., 2003). It should be emphasized that while a characteristic profile for WS is indicated by the literature, considerable variability within the cognitive and medical aspects of the profile alike have been reported (Morris et al., 1988; Udwin and Yule, 1991; Greer et al., 1997).

The vast majority of behavioral studies of people with WS describe a gregarious and socially outgoing personality type yet social difficulties are also characteristically seen (see Mervis and Klein-Tasman, 2000 for a review). Individuals with WS have been described as being less hesitant to interact with strangers than other children with developmental delays (Mervis et al., 2003), 
as well as overly friendly and affectionate (Tomc et al., 1990). The presence of these overfriendly personality traits and the perception of preserved social functioning have often led to WS being compared with autism spectrum disorders (ASDs; Rapin and Tuchman, 2008), as a contrast to the severe reciprocal social impairment characteristic of ASD (American Psychiatric Association, 2000). However, a growing body of research, summarized and discussed in the following paragraphs, has begun to suggest that social skill difficulties are present in WS, such that comparisons with ASD may be less than optimal for contributing to advances in the neuroscience of social functioning unless a more nuanced approach is taken. The present study aims to investigate the social cognitive difficulties of individuals with WS (using a lab-based measure of social cognition) that may contribute to socio-communicative and reciprocal social interaction difficulties reported by parents.

Results of studies using parent- or caregiver-completed questionnaires have revealed difficulties in various aspects of social functioning in WS. For example, Laws and Bishop (2004) reported that parents consistently rated their children with WS as performing worse on measured aspects of relationship building when compared to healthy children or those with Downs syndrome (DS) or specific language impairment (SLI; e.g., inappropriate initiation of conversation, use of stereotyped conversation). In a similar study using the same measure, children with WS showed stronger functioning than those with ASDs (Philofsky et al., 2007). In terms of social skills, parents and teachers report that children with WS typically demonstrate prosocial skill levels (e.g., cooperation, assertion, seeking out interaction) within the low average range, with more pronounced difficulty with various aspects of social functioning and social cognition apparent (Klein-Tasman et al., 2011). Studies of older individuals with WS suggest that social difficulties persist into adulthood. Generally, adults with WS are found to experience trouble making and sustaining relationships despite their tendency to be socially disinhibited and overly friendly (Udwin, 1990; Davies et al., 1998). It seems as if individuals with WS are generally interested in making friends and driven to be socially accepted but lack the understanding of social rules that would allow for successful relationships. Unfortunately, these social difficulties may become more severe with development and represent the most consistent and pervasive difficulties seen in WS (Howlin et al., 1998).

Direct observations of social interactions in individuals with WS have also revealed difficulties. Delays in the use and comprehension of pointing gestures have been observed both by parent report and in structured laboratory settings (Singer Harris et al., 1997; Laing et al., 2002). Eye gaze differences have also been reported in WS, including an interest in faces that often interferes with completion of a task presented to the child (Jones et al., 2000). Young children with WS spend more time looking at faces of social partners than do typically developing children and the quality of the gaze is often described as "intense" (Mervis et al., 2003). Preschool aged children with WS have been shown to lack social regulation. Parents report that they know no stranger, and they are more willing to approach a stranger than typically developing children of the same chronological and mental age (Dodd et al., 2010). Young children with WS are also impaired in joint attention behaviors, both in terms of initiation of and response to joint attention bids (Laing et al., 2002). Although young children with WS appear to be more responsive to displays of emotion in comparison to other children with developmental delays, this increased responsiveness does not necessarily translate to an advantage in the ability to respond adaptively in ways that are congruent with the emotions expressed (Fidler et al., 2007). Difficulties in interactions with others, such as less turn-taking with partners (Lacroix et al., 2007) and a failure to completely answer questions or provide clarifications (Stojanovik et al., 2001; Stojanovik, 2006), are also often observed.

Recent studies using a measure specifically designed to investigate difficulties in reciprocal social interaction in ASD, the Autism Diagnostic Observation Schedule (ADOS; Lord et al., 1999), have contributed to the growing understanding of social impairment in WS. Using the ADOS, Klein-Tasman et al. (2007) recently reported that approximately half of the children they examined (with limited language) exhibited abnormalities in the use of various social interactive behaviors, including eye gaze, pointing behaviors, both initiation and response to joint attention, integration of eye gaze with communicative behaviors, and reciprocal social smiling. Abnormalities in play behavior and repetitive and restricted interests were also apparent in many of these children. Further, when compared to children with developmental delays of mixed etiology (ME) and children with Pervasive Developmental Disorder-Not Otherwise Specified (PDD-NOS), many young children with WS display a behavioral profile that indicates social difficulties above and beyond what would be expected from developmental delay alone (Klein-Tasman et al., 2009).

Although social difficulties in WS have been well-documented, findings of investigations into cognitive processes related to social functioning in WS have been inconsistent and inconclusive. For example, findings in the area of face processing are mixed, with some studies concluding that individuals with WS use unique strategies to process faces (Deruelle et al., 1999; Gagliardi et al., 2003), while others find contradictory support for the use of typical strategies (Tager-Flusberg et al., 2003). Similarly, findings related to eye movement patterns while visually processing faces differ depending on the specific aspect investigated. After initially fixating in a typical fashion, individuals with WS demonstrate a decreased ability to disengage attention and spend more time looking at faces when compared to healthy controls (Riby and Hancock, 2008, 2009a). Interestingly, this difference in gaze patterns was observed when stimuli were static, but not when they were active (Riby and Hancock, 2009b), demonstrating the importance of stimulus choice. Despite a tendency to look longer at faces, individuals with WS demonstrate decreased physical arousal while viewing faces when compared to typically developing groups (Doherty-Sneddon et al., 2009). This is consistent with a complementary finding in which individuals with WS were reported to perceive physical situations as more threatening than social situations (Dodd and Porter, 2010). Investigations into theory of mind abilities in WS do not yield straightforward findings. Adults with WS seem to outperform individuals with other developmental delays on some tasks (Karmiloff-Smith et al., 1995; Tager-Flusberg et al., 1998), while children perform both similarly to and worse than comparison groups on others (Tager-Flusberg and Sullivan, 2000). Generally, people with WS have difficulties on these social 
cognitive tasks in comparison to TD controls, but it is unclear whether these difficulties are above and beyond what would be expected based on intellectual disability alone.

Task variability and diversity of contrast groups may contribute to the disparate findings in the literature, but it is clear from the discrepancies across studies that findings about social cognition in WS do not paint a straightforward picture. As is the case for both cognitive functioning and medical findings (Morris et al., 1988), there appears to be considerable variability in reciprocal social functioning within the WS population, with some, but not all individuals with WS demonstrating elevated social reciprocity difficulties. For example, Klein-Tasman et al. (2007) reported that half the children in their sample had elevated levels of social reciprocity difficulties, while half either showed very subtle social reciprocity difficulties or did not show any clear social reciprocity difficulties. The picture is further complicated by the lack of studies investigating the concordance between caregiver-reported behavior in everyday contexts and performance on tasks completed in a structured laboratory setting. Generally, studies have used either questionnaire or observational/experimental methodologies, rather than combining these approaches. Consistent findings across measures also constitute within-study replications of observations and build confidence in the reliability and validity of findings. Investigations examining the convergence between multiple methods of assessing a specific phenomenon contribute to the ecological validity of research conclusions. Finally, this research contributes to the literature exploring potential social information processing mechanisms for the social difficulties observed in children with WS; such studies of this nature may reveal target areas for intervention.

One measure of social information processing that has been used in the ASD literature but has not yet been used with children with WS is the Social Attribution Task (SAT; Klin, 2000). This task, adapted from Heider and Simmel's (1944) silent movie in which geometric shapes enact a social scene, measures the ability to attribute social meaning to a visually presented ambiguous animation. This task calls for inference of emotions, intentions, the nature of interpersonal interactions, and outcomes of interactions by anthropomorphizing the stimuli and reading non-verbal social cues. In Heider and Simmel's (1944) original description, they reported that all but one of the typically developing individuals who completed the task attributed human behaviors and emotions to the stimuli. Klin's coding scheme uses a number of indices, which are combined in order to provide a picture of broad social cognition (Klin, 2000, p. 836). More recent research using typically developing samples showed that completion of the SAT activated brain regions commonly implicated in social information processing (Schultz et al., 2003). In clinical samples, performance on the SAT was able to discriminate populations with documented social difficulties (i.e., ASDs and Prader-Willi Syndrome, PWS) from those without social impairments (Klin, 2000; Koenig et al., 2004; Klin and Jones, 2006). The video was described as "more meaningful to the normal control group, allowing [them] to generate fairly elaborated and lengthier social plots" (Klin, 2000, p. 839). These findings indicate that the SAT is an effective measure of social cognition, or more specifically the ability to attribute social meaning, including inference of common social interaction patterns and emotions, to seemingly ambiguous stimuli. Together these findings imply that this measure may be useful to further understanding social cognitive functioning in WS as well.

The current study sought to address gaps in the literature by examining the relations between social cognition and parent ratings of social reciprocity and social communication. Parent report of reciprocal social behaviors outside the laboratory setting was collected and individuals completed a lab-based measure of social information processing, the SAT (Klin, 2000). Parent ratings were related to performance on the SAT in order to investigate the concordance across measurement methodologies and to point to potential social information processing difficulties that may contribute to social reciprocity difficulties. The SAT has previously been used in individuals with ASD and was chosen based on the existing literature demonstrating its ability to differentiate clinical samples from one another (Klin, 2000; Koenig et al., 2004; Klin and Jones, 2006; see Materials and Methods for a more in-depth discussion), as well as its relation to activation in typically developing individuals of brain areas related to social cognitive processes (Schultz et al., 2003). We hypothesized that socio-communicative difficulties reported by parents would be associated with greater atypical social cognition, and weaker cognitive ability, especially verbal ability, would be associated with greater difficulty completing a laboratory based task of social cognition. We also considered the possibility that, given the intellectual disability commonly seen in WS, additional structure and support when completing the SAT, in the form of direct questioning, could be beneficial. We therefore created a direct measure of improvement within the SAT coding procedure, the Improvement Index, to quantify the difference in the quality of the narratives produced with prompting from those produced spontaneously. Using this index (further described later), exploratory analyses of the role of additional scaffolding on social attributions were conducted and related to overall intellectual functioning in order to further explore the role that cognitive abilities played in completion of the SAT.

\section{MATERIALS AND METHODS PARTICIPANTS}

The sample included 24 children with WS between the ages of 8 years 1 month and 15 years 9 months $(M=12$ years 5 months, $\mathrm{SD}=2$ years 8 months; 12 male, 12 female). Twenty-three female caregivers (22 mothers and 1 grandmother) and 1 father completed the questionnaires. Participants were recruited to participate in a study of cognitive and psychosocial functioning during the transition to adolescence. Participants were recruited by mailing fliers to families with children in the target age range through the Williams Syndrome Association, and by placing a description of the study in the registration materials at the National Williams Syndrome Convention. Note that this sample is a subset of the sample reported in Klein-Tasman et al. (2011).

\section{MATERIALS}

\section{Standardized intelligence measure}

Kaufman brief intelligence test, 2nd edition (KBIT-2). The KBIT-2 is a standardized measure of verbal and non-verbal intelligence for use with individuals ages $4-90$ years. The verbal 
intelligence scale (VIQ), which consists of two tasks measuring receptive and expressive language skills, is a measure of crystallized intelligence; the non-verbal scale (NVIQ), which involves solving visual puzzles, is a measure of fluid intelligence (Kaufman and Kaufman, 2004). Not only is this instrument one of the most commonly used brief estimates of intelligence, its use with individuals with WS is common as it does not include a spatial component, a set of skills that are often impaired in this population and therefore disproportionately affects IQ estimates (Mervis et al., 1999).

\section{Experimental measure}

Social Attribution Task. The SAT is a lab-based measure of social cognition utilizing ambiguous visual stimuli (Klin, 2000). Completing the SAT involves watching a silent video display, approximately $50 \mathrm{~s}$ long, two times through and providing a narrative summarizing the video. The individual is then shown shorter clips of the 50-s video and asked to narrate the clips separately. Finally, the individual is asked specific questions about the video. The ambiguous stimuli in the video are shapes (a small circle, a small triangle, and a large triangle) with no faces or other features similar to humans or other animals. These shapes move around the screen throughout the duration of the video. A more detailed description of instructions and prompts used in the SAT administration is included in the Section "Procedure."

\section{Parent report measures}

Social communication questionnaire (SCQ). The SCQ is a 40item parent questionnaire for use with children ages 4 and older. The responses that caregivers provide about their children's social communication behaviors yield a total score. Scores above 15 points indicate social-communication difficulties that warrant further assessment for the presence of an ASD. This questionnaire is meant to serve as an efficient method of identifying children with communication and social delays (Rutter et al., 2003).

Social reciprocity scale (SRS). The SRS is a 65-item parent questionnaire for use with children ages 4-18 years used to explore symptoms of ASDs, including difficulties in interpersonal relationships, communication, and repetitive/stereotypic behaviors. Not only is the identification of these symptoms useful when screening for ASDs in particular, but can also be helpful in identifying individuals with problem behaviors in these domains that are at subthreshold levels. The responses that caregivers provided about their children's social reciprocity behaviors yielded $T$-scores on various scales of the SRS. These include the Social Awareness, Social Cognition, Social Communication, Social Motivation, and Autistic Mannerisms scales, as well as an overall total score. $T$-scores below 60 indicate no clinically significant concerns in social reciprocity behaviors; $T$-scores of $60-75$ indicate social reciprocity difficulties that are in the mild to moderate range; $T$ scores greater than 76 indicate severe levels of social reciprocity difficulties (Constantino and Gruber, 2005).

\section{PROCEDURE}

\section{DATA COLLECTION}

Children participated either at the Child Neurodevelopment Research Lab (CNRL) at the University of Wisconsin, Milwaukee, in a quiet location at their homes, or in a quiet location at a Williams Syndrome Association Bi-Annual Meeting. All children were administered a battery of assessment measures, including a standardized measure of intelligence (KBIT-2) and other standardized and lab-based measures, including the SAT, over the course of an approximately $2 \mathrm{~h}$ long session. Parents of children completed questionnaires and interviews regarding anxiety symptoms and adaptive functioning either in a separate room or at a different time. All components of the child assessment were videotaped to review the procedure and allow for transcription of responses to the SAT.

\section{SAT administration and coding procedures}

Administration and subsequent coding procedures for the SAT followed those described by Klin's (2000). All administrations of the SAT were initially transcribed from videotape by an undergraduate research assistant in the CNRL and then reviewed by the author for accuracy. The first author and two research assistants initially coded four administrations and agreement between coders was measured using correlational statistics. Disagreements were discussed in order to improve reliability. Another four administrations were then coded and agreement between coders was measured. At this point, agreement was acceptable (Pertinence Index $r=0.913$, Salience Index $r=0.866$, Theory of Mind Cognition Index $r=0.980$, Theory of Mind Affect Index $r=0.908$, Animation Index $r=0.718$, Problem Solving A Index $r=0.982$, Problem Solving B Index $r=0.967$, Improvement Index $r=0.993)$. The first author then coded all transcripts and the two research assistants each coded a portion of the transcripts, resulting in each administration being coded twice. Consensus coding was then conducted in order to resolve any disagreements and arrive at a single value for each index.

The Salience, Pertinence, and Animation indices, as well as the Theory of Mind Cognition and Affect indices were coded. See Table 1 for a brief overview of coding procedures information. The Salience Index is a reflection of the individual's ability to make a coherent social story from visual information that fits in with what the majority of other individuals see in the same task. It serves as an estimation of the individual's overall ability to view the ambiguous stimuli and extract social information from what they see. In a real world setting, this would be similar to a situation in which an individual must make decisions about the behaviors of others and determine their meaning within a social context. The Pertinence Index is a measure of an individual's ability to make attributions that reflect relevance to the viewed stimuli. It is an estimation of the individual's ability to view stimuli and extract relevant information; this ability relates to real world social functioning, in which individuals need to determine exactly what information is socially relevant. The Animation Index is similar to a summary measure of social attribution; it reflects an estimation of the individual's overall level of social cognitive ability. Two Theory of Mind indices, Cognition and Affect, were also included in this investigation. These indices measure the frequency of references to cognitive or affective mental states, reflecting attention to the thoughts and feelings of others, a critical component when discussing the construct of theory of mind and understanding the nature of social interactions in general. 
Table 1 | Overview of coding procedures.

\begin{tabular}{|c|c|c|c|}
\hline Index & Narratives & Description & Measurement \\
\hline Pertinence & $1-7$ & Ratio of non-pertinent statements to total propositions & $0.00-1.00$ \\
\hline Salience & $1-7$ & Percentage of correctly identified story elements out of 20 salient story elements & $\%$ \\
\hline Theory of mind - cognitive & $1-7$ & $\begin{array}{l}\text { Ratio of number of statements indicating thinking, planning, or intentionality to total } \\
\text { propositions }\end{array}$ & $0.00-1.00$ \\
\hline Theory of mind - affective & $1-7$ & Ratio of number of statements indicating feeling states to total propositions & $0.00-1.00$ \\
\hline Animation & $1-7$ & Ordinal rating of sophistication of social cognition & $0-6$ \\
\hline Problem solving & $11-17$ & Percentage of questions answered correctly & $\%$ \\
\hline
\end{tabular}

Adapted from Koenig et al. (2004).

In addition to these indices, reported in Klin's original investigation, a novel index, the Improvement index was also coded to investigate the effect of providing greater structure during completion of the SAT. SAT administration guidelines involve first having the respondent describe what is seen with minimal prompts, and later specific prompts are provided to elicit elaboration. Arriving at a score for the Improvement index involves coding the Problem Solving index twice. The first time it is scored using the spontaneous answers the individual provides to open-ended questions during the initial administration of the SAT. It is then scored again using the answers the respondent provides in response to the more directed questions the examiner asks. For example, when first completing the administration, the examiner simply asks "What happened here?" after each clip is shown. However, when specific instructions are given, the examiner says, "Now let's say that the big triangle, the small triangle, and the circle are people. What kind of person is the big triangle? the small triangle? the circle?" The difference in the number of "correct" answers the individual provides in these two situations is then the score for the Improvement index.

\section{RESULTS}

All analyses were conducted using SPSS computing software. Significance tests were 2 -tailed. It should be noted that all 24 children completed the intelligence measure and the SAT, while 21 had parent questionnaire data. All available data were used for each analysis.

\section{INTELLECTUAL ABILITIES}

The average overall IQ composite standard score, as measured with the KBIT-II, was 65.71 $(\mathrm{SD}=11.99)$, with verbal and non-verbal IQ not significantly different from one another $(M=73.08, \mathrm{SD}=11.96$ and $M=66.29, \mathrm{SD}=13.56$, respectively). These results indicate that, on average, the current sample's intellectual functioning is falling in the mildly impaired range, which is consistent with the level of functioning and range seen in individuals with WS in other studies.

\section{SOCIAL RECIPROCITY}

Average $T$-scores and SDs for each domains are reported in Table 2. The number of children with $T$-scores falling within different classifications on the various domains is reported in Figure 1. The pattern of results indicates that on average, the
Table 2 | Parent-reported social reciprocity skills.

\begin{tabular}{lll}
\hline SRS domain & Mean $\boldsymbol{T}$-score & SD \\
\hline Social awareness & 64.48 & 11.17 \\
Social cognition & 76.05 & 11.29 \\
Social communication & 66.86 & 10.55 \\
Social motivation & 55.24 & 15.92 \\
Autistic mannerisms & 75.05 & 15.10 \\
Total score & 70.24 & 11.17
\end{tabular}

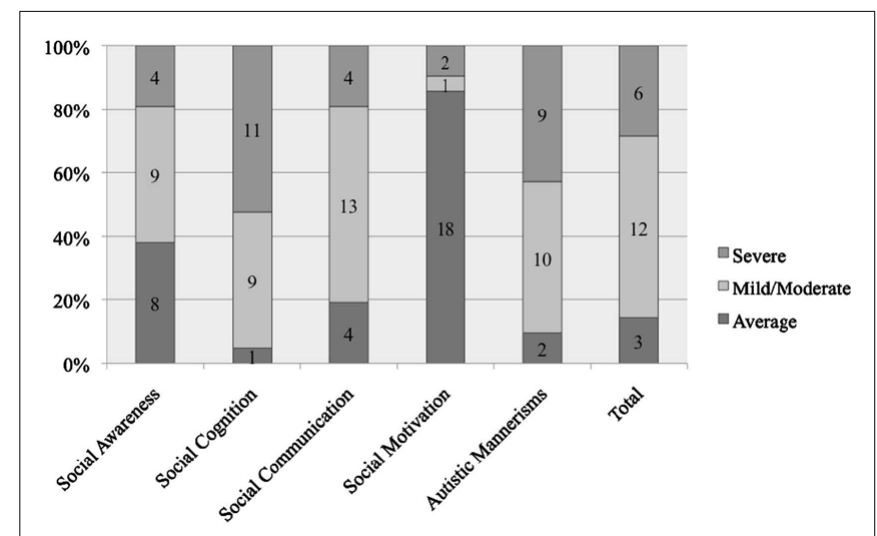

FIGURE 1 | Percent of participants falling in the average, $\mathrm{mild} /$ moderate, and severe ranges on the social responsiveness scale parent report measure (number of participants indicated).

children in this sample had mild to moderately elevated total scores, with the most severe difficulties in the area of Social Cognition and average range scores in the Social Motivation domain. Further discussion of these findings, demonstrating clear social reciprocity difficulties and good concordance between parent and teacher ratings, can be found in a separate publication from our lab (Klein-Tasman et al., 2011).

\section{SOCIAL COMMUNICATION}

The average score on the SCQ in this population was 11.75 $(\mathrm{SD}=6.39)$; however, what is more meaningful when interpreting this particular questionnaire is the number of children who met or exceeded the cutoff score of 15 and the relationship between meeting this cutoff and SAT performance. The distribution of scores on 
the SCQ was continuous. In this sample, seven children (35\%) met or exceeded the cutoff score, which indicates a need for additional screening for ASDs.

\section{SOCIAL COGNITION}

Mean scores for each of the indices of the SAT are reported in Table 3. Normative data are not available. The mean number of propositions spontaneously supplied in Narratives 1 through 7 was $20.71(\mathrm{SD}=9.72)$. This number is generally similar to those reported in previous studies using clinical samples (Klin, 2000; Koenig et al., 2004). Table 4 provides examples of different quality narratives supplied by participants in the current study.

\section{RELATIONS BETWEEN SCO AND SRS}

Statistically significant and strong correlations were found between the total score of the SCQ and all domains of the SRS (Social Awareness $r=0.768, p<0.01$; Social Cognition $r=0.707$, $p<0.01$; Social Communication $r=0.652, p<0.01$; Autistic Mannerisms $r=0.702, p<0.01$; total $r=0.837, p<0.01)$, with the exception of the Social Motivation domain $(r=0.375$, ns). Given the outgoing nature of individuals with WS, as well as previous findings that individuals with WS do not have difficulty in the social motivation domain (Klein-Tasman et al., 2011), a weak correlation between the total score of the SCQ and the Social Motivation domain of the SRS was not unexpected.

Table 3 | Social Attribution Task (SAT) index scores.

\begin{tabular}{lll}
\hline SAT index & Mean & SD \\
\hline Pertinence & 0.36 & 0.30 \\
Salience & 3.33 & 2.62 \\
Theory of mind - cognitive & 0.06 & 0.12 \\
Theory of mind - affect & 0.03 & 0.06 \\
Animation & 1.46 & 0.72 \\
Problem solving & 0.21 & 0.19 \\
Improvement & 2.13 & 1.87 \\
\hline
\end{tabular}

Table 4 | Sample narratives.

\section{RELATIONS BETWEEN PERFORMANCE ON THE SAT AND INTELLECTUAL FUNCTIONING}

Significant relations were found for the Pertinence index and VIQ $(r=-0.437, p<0.05)$ and the Problem Solving index and VIQ $(r=0.446, p<0.05)$, NVIQ $(r=0.416, p<0.05)$, and IQ composite score $(r=0.544, p<0.05)$. The Improvement index was also significantly related to VIQ $(r=0.398, p<0.05)$, NVIQ $(r=0.578, p<0.01)$, and IQ composite score $(r=0.628$, $p<0.001)$. There were no significant relations found for the Salience, Theory of Mind: Cognition, Theory of Mind: Affect, or Animation indices and score on the KBIT-II.

\section{RELATIONS BETWEEN PERFORMANCE ON THE SAT AND AGE}

There was a significant relation between age and performance on the Salience $(r=0.403, p<0.05)$, Theory of Mind: Affect $(r=0.402, p<0.05)$, and Problem Solving $(r=0.448, p<0.05)$ indices. There was no significant relation between the age at which the SAT was administered and performance on the Pertinence, Theory of Mind: Cognition, Animation, or Improvement indices.

\section{RELATIONS BETWEEN PERFORMANCE ON THE SAT AND PARENT-REPORTED SOCIAL RECIPROCITY}

Significant relations between indices and $T$-scores on the domains of the SRS are reported in Table 5. All significant relations were negative, indicating that as scores on the SRS increased (suggestive of greater difficulty), scores on the SAT decreased (suggestive of greater difficulty). Scores on the Theory of Mind: Cognition Index were related to the Autistic Mannerisms scale. Scores on the Salience Index were significantly correlated with the Social Cognition, Social Communication, Social Motivation, and Autistic Mannerisms scales, as well as the overall total score. Scores on the Animation index were related to the Social Awareness scale and the SRS Total score. Scores on the Problem Solving Index were significantly correlated with the Social Awareness, Social Communication, and Autistic Mannerisms domain scores and the total score. Finally, scores on the Improvement Index were significantly related to the Social Awareness domain score. There were no significant relations found for the Pertinence and Theory of Mind: Affect indices of the SAT and any of the scales of the SRS.

Higher quality narratives The smaller triangle and the circle came and went inside and was having fun and the triangle went out and was playing tag with the smaller one and then the circle kind of shut the door and the little one opened it and then the other one was still outside having fun and then the smaller circle and then the smaller triangle went out and the bigger triangle shut the box and destroyed it.

What happened was like that triangle went through the triangle, I mean the square, and up... and all of the sudden the circle finally came in and closed the door on him and the triangle was stuck for a minute and the triangle got out and the triangle friend came in ... so they were um both in the house and the triangle went out and slammed the door behind him so they were looking for each other and stuff and they came around and like together like walking and all of a sudden the triangle starts to chase him so the triangle and the circle go running across the screen and out of the picture and the triangle messes up the square kind of and that was it.

Lower quality narratives I saw a circle, a square and a triangle. Sometimes umm the triangle will umm bump into another triangle and then sometimes the circle would bump into the triangle and sometimes the square would open and close and then umm at the end I saw two lines and that's it.

... the big triangle chased the little triangle and the little ball goes there and then the triangle chased the triangle and the ball and then they went back that way and then went plew, right through it and that was it. 
Table 5 | Correlations between SAT performance and parent-reported social reciprocity skills.

\begin{tabular}{|c|c|c|c|c|c|c|}
\hline \multirow[t]{2}{*}{ SAT index } & \multicolumn{6}{|c|}{ Social responsiveness scale domain } \\
\hline & $\begin{array}{l}\text { Social } \\
\text { awareness }\end{array}$ & $\begin{array}{l}\text { Social } \\
\text { cognition }\end{array}$ & $\begin{array}{l}\text { Social } \\
\text { communication }\end{array}$ & $\begin{array}{l}\text { Social } \\
\text { motivation }\end{array}$ & $\begin{array}{l}\text { Autistic } \\
\text { mannerisms }\end{array}$ & Total score \\
\hline Pertinence & 0.383 & 0.269 & 0.375 & 0.238 & 0.197 & 0.377 \\
\hline Salience & -0.342 & $-0.636^{* *}$ & $-0.615^{* *}$ & $-0.466^{*}$ & $-0.534^{* *}$ & $-0.640^{* *}$ \\
\hline Theory of mind - cognition & -0.143 & -0.102 & -0.191 & -0.206 & $-0.432^{*}$ & -0.268 \\
\hline Theory of mind - affect & 0.059 & -0.374 & -0.300 & -0.194 & -0.381 & -0.226 \\
\hline Animation & $-0.459 *$ & -0.269 & -0.339 & -0.299 & $-0.528 * *$ & $-0.492^{*}$ \\
\hline Problem solving & $-0.624^{* *}$ & -0.365 & $-0.487^{*}$ & -0.249 & $-0.514^{*}$ & $-0.547^{* *}$ \\
\hline Improvement & $-0.480^{*}$ & -0.100 & -0.225 & -0.108 & -0.259 & -0.272 \\
\hline
\end{tabular}

$* p<0.05$.

$* * p<0.01$.

Given the significant relations with intellectual functioning, partial correlations to examine the relationships between SAT indices and the SRS taking into account KBIT-2 IQ were conducted. Significant negative relationships remained between performance on the Salience index and the Social Cognition $(r=-0.613, p<0.01)$, Social Communication $(r=-0.561$, $p<0.01)$, Social Motivation $(r=-0.458, p<0.05)$, and Autistic Mannerisms scales $(r=-0.528, p<0.01)$ and the SRS total score $(r=-0.602, p<0.01)$. Negative relationships between the Animation index and the Autistic Mannerisms scale $(r=-0.515$, $p<0.05)$ and the Problem Solving Index and the Social Awareness $(r=-0.490, p<0.05)$ and Autistic Mannerisms $(r=-0.479$, $p<0.05)$ scales remained significant as well.

\section{RELATIONS BETWEEN PERFORMANCE ON THE SAT AND PARENT-REPORTED IMPAIRMENTS ON A SCREENING MEASURE FOR ASD}

Significant relations were found for the Salience Index and total score of the SCQ $(r=-0.457, p<0.05)$, as well as the Animation Index and total score on the SCQ $(r=-0.493$, $p<0.05)$ and classification on the SCQ $(r=-0.495, p<0.05)$. The Problem Solving index was also significantly related to total score on the SCQ $(r=-0.596, p<0.05)$ and classification on the SCQ $(r=-0.480, p<0.05)$. There were no significant relations found for the Pertinence, Theory of Mind Cognition, Theory of Mind Affect, or Improvement indices of the SAT and score on the SCQ. When intellectual functioning was taken into account using partial correlations, significant relations between SCQ classification and the Animation index $(r=-0.455, p<0.05)$ and the Problem Solving index $(r=-0.477, p<0.05)$ remained. The negative direction of these relationships indicates that as scores on the SCQ increase, reflecting greater difficulty, scores on the SAT decrease, also reflecting greater difficulty.

\section{CHANGE IN SAT PERFORMANCE WITH THE ADDITION OF SPECIFIC INSTRUCTIONS}

Participants were first asked to describe the scenes in the video without specific instructions; on average, only $4.17 \%$ of the "target" answers were spontaneously provided during these narratives.
However, when the participants were given instructions as to how to view the stimuli, $21.25 \%$ of the "target" answers were provided. These instructions included directions as to how to view the shapes in the video (i.e., as people) and how to interpret the interactions they had (see Klin, 2000 for specific questions). In other words, when the individuals in this sample were provided with the additional specific instructions that are part of the SAT administration process about how to interpret the traits and actions of the previously ambiguous stimuli as socially meaningful, there was a significant change in the quality of the narratives they were able to provide $[t(23)=4.833$, $p<0.005]$. This improvement that was seen with the additional scaffolding was significantly related to overall level of intellectual functioning $(r=0.628, p<0.01)$, such that those with stronger intellectual functioning showed larger improvements with scaffolding.

\section{DISCUSSION}

Both questionnaire and laboratory based studies have revealed difficulties in social reciprocity in children and adults with WS. However, few studies have used multiple converging measures in the same individuals to evaluate the relationship between informant report and observable behaviors seen in a laboratory setting and to explore the social information processing difficulties that may contribute to social reciprocity challenges. The goal of the current study was to carry out such an examination using a lab-based measure of social cognition and intellectual and parent-rated socio-communicative and social reciprocity functioning in children with WS. As hypothesized, results indicated a significant relationship between directly measured social processing abilities and reciprocal social behaviors in WS children as rated by parents. Intellectual functioning and social cognition were also found to be significantly related, however the modest strength of this relationship suggested that intellectual functioning alone does not explain SAT performance. Furthermore, significant relationships between social processing and reciprocal social behaviors remained after accounting for intellectual ability, suggesting that difficulties in social cognition have a unique role in the social reciprocity difficulties of individuals with WS. 


\section{RELATIONS BETWEEN SOCIAL COGNITION AND PARENT-REPORTED BEHAVIOR}

Individuals with WS who were more adept at making social attributions were also rated higher in terms of parent-reported social reciprocity skills, motivation to engage in social activities, the ability to interpret social cues in the world, and the level of expressive social communication. Similarly, individuals with WS who provided answers that were more consistent with those provided by typically developing adolescents and adults (as reflected by higher ratings on the Problem Solving index) were rated as more aware of social cues in the real world. Essentially, individuals with WS who made more appropriate social attributions in this lab-based task were also rated by their parents as more socially aware and competent in their daily lives. This relationship is further supported by the findings of a strong correlation between the two parent report measures used and their similar relationship to the SAT. Specifically, both SCQ and SRS scores were correlated with the Animation and Problem Solving indices of the SAT, which are both reflections of common social interpretations of the ambiguous scenes.

As past reports have indicated, a proportion of individuals with WS have an interest in others while simultaneously lacking the appropriate skills necessary to sustain interactions and form lasting relationships (Davies et al., 1998; Laws and Bishop, 2004). The consistency and pervasiveness of this difficulty with relationships is actually one of the most frequently reported concerns of caregivers (Udwin, 1990). Based on studies using questionnaires or lab-based measures separately, individuals with WS have difficulty comprehending environmental cues that are important to social functioning, such as non-verbal aspects of language, or pragmatics (Philofsky et al., 2007) and perspective taking (Fidler et al., 2007). Klein-Tasman et al. (2011) found that while children with WS were reported to have social reciprocity difficulties, they were more related to difficulties in social cognition than in social motivation. These difficulties likely contribute to the decreased ability to establish and maintain meaningful relationships, despite superficially average overt social initiation skills. The results of the current study support the assertion that individuals with more pronounced social difficulties as reported by caregiver questionnaire are also more likely to have difficulties picking up on the typical social information relevant to social scenes, such as the various roles played by those participating in an interaction, the potential feelings and subsequent motivations for actions, and the consequences of those actions. When people with WS do not pick up on these aspects of others' social behavior, this likely contributes to difficulties with successful social interactions.

\section{FACILITATIVE EFFECTS OF ADDITIONAL STRUCTURE}

An additional exploratory aim was to examine the effect of additional structure on social attributions made by the participants. The vast majority of participants in this study were unable to produce narratives that spontaneously correctly answered even one of the obvious questions related to the stimuli. However, when asked directly about these aspects, participants were more able to provide answers that were consistent with previously identified normative answers (Klin, 2000). Moreover, the beneficial effects of specific questioning were more pronounced for participants with stronger intellectual functioning. The additional structure provided by asking specific questions is similar to the concept of scaffolding, a metaphor first discussed at length by Wood et al. (1976). Similarly to Vygotsky's zone of proximal development (Vygotsky, 1978), scaffolding refers to the structure and additional instruction that parents, teachers, and other caregivers provide when children are attempting to complete a task with components that are not yet at the level of mastery (Stone, 1998). The benefits of scaffolding for both typically developing children and those with developmental delays are widely acknowledged and studied. Specifically, the use of scaffolding has been shown to aid children with delays in the acquisition of language (Kirchner, 1991), the development of social skills (Baker et al., 2007), and in improving reading comprehension abilities (Dieterich et al., 2006). The current study, which includes participants with mild to moderate intellectual impairments, demonstrates the added benefits of scaffolding while completing a social cognitive activity. A significantly greater number of target responses were produced when the participants were given more explicit instructions about how to view the stimuli.

Given the importance of effective scaffolding, parent-training programs for children with developmental delays could focus on ways in which to structure the environment to ensure consistent skill acquisition. The benefits of scaffolding in the current study fit with these findings and may suggest that these types of interventions and additional structure may provide some individuals with WS (i.e., those with less severe cognitive impairments) with the additional resources needed to more effectively interpret ambiguous social stimuli and gain skills in social reciprocity. It appears as though the children in the current study with more severe intellectual impairments do not benefit from the additional structure provided; it is possible that the ambiguous stimuli, which are shapes that do not physically resemble humans or animals, were simply too abstract for them. These limitations to the benefit of scaffolding are important to keep in mind; interventions using more abstract materials may not in fact be beneficial even with additional structure.

\section{LIMITATIONS AND FUTURE DIRECTIONS}

The current study represents the first investigation into the performance of individuals with WS on the SAT, a lab-based measure of social cognition and attribution, and relations to behavioral and intellectual characteristics. Although a typically developing control group was not included, results revealed a number of relationships between social cognition abilities and parent-reported behavior. The inclusion of control groups in future investigations would allow for exploration of questions related to the developmental trajectories (i.e., delay or deviance) of social cognition in WS and further explore the potential influence of intellectual functioning. In particular, a contrast group of individuals with ASDs would allow for comparisons to a population with documented and consistent difficulties in social cognitive and social reciprocity skills. As was demonstrated in previous studies using the SAT (Klin, 2000; Koenig et al., 2004; Klin and Jones, 2006), individuals with ASDs have difficulty completing the task in comparison to both typical and clinical control groups, demonstrating an underlying difficulty in social cognitive processing. Direct comparisons to children with ASD would allow for further specification of the aspects of reciprocal social interaction difficulties that are also 
shared by individuals with WS, and those aspects that appear to be less commonly seen in WS and differentiate WS from ASD. Regardless of the relations found, these types of investigations would provide a better understanding of the social cognitive difficulties that relate to the observable social difficulties in these two clinical populations and may even play a role in determining the presence of a comorbid ASD in individuals with WS. In addition, other contributors to performance on the SAT warrant examination. For example, it is possible that additional personality character traits, such as empathy and emotional responsivity, may contribute to SAT performance for children with WS.

The SAT was used as a way to add to the literature about social cognition in WS, and we have made some attempt to elucidate aspects of social cognition that are likely measured by the SAT. However, as is the case for other studies of social cognition, the current study is somewhat limited by a lack of a unifying theory as to what specific components contribute to skills that fall under the umbrella term "social cognition." Emotion recognition, face processing, empathy, and theory of mind abilities are just a few of the possible contributors to social information processing; a number of additional processes, including understanding of ambiguous social dynamics, also likely playing a part in social cognition and should be considered. The field would benefit from a well-defined and clearly outlined theory of social cognition that would allow for future studies to more explicitly explore mechanisms of reciprocal social behaviors and models of the relations among the various facets of social cognition.

\section{REFERENCES}

American Psychiatric Association. (2000). Diagnostic and Statistical Manual of Mental Disorders, 4th Edn, Text Revision. Washington, D.C.: American Psychiatric Association.

Baker, J. K., Fenning, R. M., Crnic, K. A., Baker, B. L., and Blacher. (2007). Prediction of social skills in 6-year-old children with and without developmental delays: contributions of early regulation and maternal scaffolding. Am. J. Ment. Retard. 112, 375-391.

Constantino, J. N., and Gruber, C. P. (2005). Social Responsiveness Scale (SRS). Los Angeles, CA: Western Psychological Services.

Davies, M., Udwin, O., and Howlin, P. (1998). Adults with Williams syndrome: preliminary study of social, emotional and behavioural difficulties. Br. J. Psychiatry 172, 273-276.

Deruelle, C., Mancini, J., Livet, M. O., Cassé-Perrot, C., and de Schonen, S. (1999). Configural and local processing of faces in children with Williams syndrome. Brain Cogn. 41, 276-298.

Dieterich, S. E., Assel, M. A., Swank, P., Smith, K. E., and Landry, S. H. (2006). The impact of early maternal verbal scaffolding and child language abilities on later decoding and

The present study demonstrated relationships between the performance of individuals with WS on a lab-based measure of social cognition and various parent-reported sociocommunicative abilities, as well as cognitive functioning. The significant relations observed point to underlying social cognitive processing difficulties that may contribute to social reciprocity behaviors observed in the natural setting outside of the laboratory, even once variability in intellectual functioning is taken into account. In addition, the results provide evidence that additional structure and support can potentially help individuals with WS, particularly those with stronger intellectual functioning, more effectively process social information. Further study of social information processing difficulties underlying the social reciprocity limitations of individuals with WS is warranted to point toward targets for effective intervention.

\section{ACKNOWLEDGMENTS}

The authors wish to thank Kristin (Phillips) Smith, Kelly Janke, Elaine Bennaton, and Emily Erdmann for assistance with data collection and coding. This research was made possible by a Center Scholar grant from the Center for Applied Behavioral Health Research at the University of Wisconsin-Milwaukee and private donations to the Child Neurodevelopment Research Lab. We are grateful to the Williams Syndrome Association for their support of research, and especially to the children and their families for participating.

young children with Williams syndrome. Am. J. Ment. Retard. 112, 194-206.

Frangiskakis, J. M., Ewart, A. K., Morris, C. A., Mervis, C. B., Bertrand, J., Robinson, B. F., Klein, B. P., Ensing, G. J., Everett, L. A., Green, E. D., Pröschel, C., Gutowski, N. J., Nobel, M., Atkinson, D. L., Odelberg, S. J., and Keating, M. T. (1996). LIMkinase1 hemizygosity implicated in imapired visuospatial constructive cognition. Cell 86, 59-69.

Gagliardi, C., Frigerio, E., Burt, D. M., Cazzaniga, I., Perrett, D. I., and Borgatti, R. (2003). Facial expression recognition in Williams syndrome. Neuropsychologia 41, 733-738.

Gosch, A., and Pankau, R. (1997). Personality characteristics and behaviour problems in individuals of different ages with Williams syndrome. Dev. Med. Child Neurol. 39, 527-533.

Greer, M. K., Brown, F. R., Pai, G. S., Choudry, S. H., and Klein, A. J. (1997). Cognitive, adaptive, and behavioral characteristics of Williams syndrome. Am. J. Med. Genet. 74, 521-525.

Heider, F., and Simmel, M. (1944). An experimental study of apparent behavior. Am. J. Psychol. 57, 243-259.
Hillier, L. W., Fulton, R. S., Fulton, L. A., Graves, T. A., Pepin, K. H., Wagner-McPherson, C., Layman, D., Maas, J., Jaeger, S., Walker, R., Wylie, K., Sekhon, M., Becker, M. C., O’Laughlin, M. D., Schaller, M. E., Fewell, G. A., Delehaunty, K. D., Miner, T. L., Nash, W. E., Cordes, M., Du, H., Sun, H., Edwards, J., Bradshaw-Cordum, H., Ali, J., Andrews, S., Isak, A., Vanbrunt, A., Nguyen, C., Du, F., Lamar, B., Courtney, L., Kalicki, J., Ozersky, P., Bielicki, L., Scott, K., Holmes, A., Harkins, R., Harris, A., Strong, C. M., Hou, S., Tomlinson, C., DauphinKohlberg, S., Kozlowicz-Reilly, A., Leonard, S., Rohlfing, T., Rock, S. M., Tin-Wollam, A. M., Abbott, A., Minx, P., Maupin, R., Strowmatt, C., Latreille, P., Miller, N., Johnson, D., Murray, J., Woessner, J. P., Wendl, M. C., Yang, S. P., Schultz, B. R., Wallis, J. W., Spieth, J., Bieri, T. A., Nelson, J. O., Berkowicz, N., Wohldmann, P. E., Cook, L. L., Hickenbotham, M. T., Eldred, J., Williams, D., Bedell, J. A., Mardis, E. R., Clifton, S. W., Chissoe, S. L., Marra, M. A., Raymond, C., Haugen, E., Gillett, W., Zhou, Y., James, R., Phelps, K., Iadanoto, S., Bubb, K., Simms, E., Levy, R., Clendenning, J., Kaul, R., Kent, W. J., Furey, T. S., Baertsch, R. A., Brent, 
M. R., Keibler, E., Flicek, P., Bork, P., Suyama, M., Bailey, J. A., Portnoy, M. E., Torrents, D., Chinwalla, A. T., Gish, W. R., Eddy, S. R., McPherson, J. D., Olson, M. V., Eichler, E. E., Green, E. D., Waterston, R. H., and Wilson, R. K. (2003). The DNA sequence of human chromosome 7. Nature 424, 157-164.

Howlin, P., Davies, M., and Udwin, O. (1998). Syndrome specific characteristics in Williams syndrome: to what extent do early behavioural patterns persist into adult life? J. Appl. Res. Intellect. Disabil. 11, 207-226.

Jones, W., Bellugi, U., Lai, Z., Chiles, M., Reilly, J., Lincoln, A., and Adolphs, R. (2000). II. Hypersociability in Williams syndrome. J. Cogn. Neurosci. 12, 30-46.

Karmiloff-Smith, A., Klima, E., Bellugi, U., Grant, J., and Baron-Cohen, S. (1995). Is there a social module? Language, face processing, and theory of mind in individuals with Williams syndrome. J. Cogn. Neurosci. 7, 196-208.

Kaufman, A. S., and Kaufman, N. L. (2004). Kaufman Brief Intelligence Test, 2nd Edn. Circle Pines, MN: AGS Publishing.

Kirchner, D. M. (1991). Using verbal scaffolding to facilitate conversational participation and language acquisition in children with pervasive developmental disorders. J. Child. Commun. Disord. 14, 81-98.

Klein-Tasman, B. P., Li-Barber, K. T., and Magargee, E. T. (2011). Honing in on the social phenotype in williams syndrome using multiple measures and multiple raters. J. Autism Dev. Disord. 41, 341-351.

Klein-Tasman, B. P., and Mervis, C. B. (2003). Distinctive personality characteristics of 8-, 9-, and 10-year-olds with Williams syndrome. Dev. Neuropsychol. 23 (1\&2), 269-290.

Klein-Tasman, B. P., Mervis, C. B., Lord, C., and Phillips, K. D. (2007). Socio-communicative deficits in young children with Williams syndrome: performance on the autism diagnostic observation schedule. Child Neuropsychol. 13, 444-467.

Klein-Tasman, B. P., Phillips, K. D., Lord, C., Mervis, C. B., and Gallo, F. J. (2009). Overlap with the autism spectrum in young children with Williams syndrome. J. Dev. Behav Pediatr. 30, 289-299.

Klin, A. (2000). Attributing social meaning to ambiguous visual stimuli in higher-functioning autism and Asperger syndrome: the social attribution task. $J$. Child Psychol. Psychiatry 41, 831-846.

Klin, A., and Jones, W. (2006). Attributing social and physical meaning to ambiguous visual displays in individuals with higher-functioning autism spectrum disorders. Brain Cogn. $61,40-53$.

Koenig, K., Klin, A., and Schultz, R. (2004). Deficits in social attribution ability in Prader-Willi syndrome. J. Autism Dev. Disord. 34, 573-582.

Lacroix, A., Bernicot, J., and Reilly, J. (2007). Narration and collaborative conversation in Frenchspeaking children with Williams syndrome. J. Neurolinguistics 20, 445-461.

Laing, E., Butterworth, G., Ansari, D., Gsödl, M., Longhi, E., Panagiotaki, G., S. Paterson, and A. KarmiloffSmith (2002). Atypical development of language and social communication in toddlers with Williams syndrome. Dev. Sci. 5, 233-246.

Laws, G., and Bishop, D. V. M. (2004). Pragmatic language impairment and social deficits in Williams syndrome: a comparison with Down's syndrome and specific language impairment. Int. J. Lang. Commun. Disord. $39,45-64$.

Lord, C., Rutter, M., DiLavore, P., and Risi, S. (1999). Autism Diagnostic Observation Schedule (ADOS) Manual. Los Angeles, CA: Western Psychological Services.

MacDonald, G. W., and Roy, D. L. (1988). Williams syndrome: a neuropsychological profile. J. Clin. Exp. Neuropsychol. 10, 125-131.

Mervis, C. B., and Bertrand, J. (1997). "Developmental relations between cognition and language: evidence from Williams syndrome," in Communication and Language Acquisition: Discoveries from Atypical Development, eds L. B. Adamson and M. A. Romski (New York: Brookes), 75-106.

Mervis, C. B., and Klein-Tasman, B. P. (2000). Williams syndrome: cognition, personality, and adaptive behavior. Ment. Retard Dev. Disabil. Res. Rev. 6, 148-158.

Mervis, C. B., Morris, C. A., KleinTasman, B. P., Bertrand, J., Kwitny, S., Applebaum, L. G., and Rice, C. E. (2003). Attentional characteristics of infants and toddlers with Williams syndrome during triadic interactions. Dev. Neuropsychol. 23, 243-268.
Mervis, C. B., and Robinson, B. F. (2000). Expressive vocabulary ability of toddlers with Williams syndrome or down syndrome: a comparison. Dev. Neuropsychol. 17 111-126.

Mervis, C. B., Robinson, B. F., Bertrand, J., Morris, C. A., Klein-Tasman, B. P., and Armstrong, S. C. (2000). The Williams syndrome cognitive profile. Brain Cogn. 44, 604-628.

Mervis, C. B., Robinson, B. F., and Pani, J. R. (1999). Visuospatial Construction. Am. J. Hum. Genet. 65 1222-1229.

Morris, C. A., Demsey, S. A., Leonard, C. O., Dilts, C., and Blackburn, B. L. (1988). Natural history of Williams syndrome: physical characteristics. $J$. Pediatr. 113, 318-326.

Morris, C. A., Mervis, C. B., Hobart, H. H., Gregg, R. G., Bertrand, J., Ensing, G. J., Sommer, A., Moore, C. A., Hopkin, R. J., Spallone, P. A., Keating, M. T., Osborne, L., Kimberley, K. W. and Stock, A. D. (2003). GTF2I hemizygosity implicated in mental retardation in Williams syndrome: genotype-phenotype analysis of five families with deletions in the Williams syndrome region. Am. J. Med. Genet. A 123A, 45-59.

Osborne, L. R., Campbell, T., Daradich, A., Scherer, S. W., and Tsui, L. (1999). Identification of a putative transcription factor gene (WBSCR11) That is commonly deleted in Williams-Beuren syndrome. Genomics 57, 279-284.

Philofsky, A., Fidler, D. J., and Hepburn, S. (2007). Pragmatic language profiles of school-age children with autism spectrum disorders and Williams syndrome. Am. J. Speech Lang. Pathol. 16, 368-380.

Rapin, I., and Tuchman, R. F. (2008). Autism: definition, neurobiology, screening, diagnosis. Pediatr. Clin. North Am. 55, 1129-1146.

Riby, D. M., and Hancock, P. J. B. (2008). Viewing it differently: social scene perception in Williams syndrome and Autism. Neuropsycholgia 46, 2855-2860.

Riby, D. M., and Hancock, P. J. B. (2009a). Do faces capture the attention of individuals with williams syndrome or autism? Evidence from tracking eye movements. J. Autism Dev. Disord. 39, 421-431.

Riby, D. M., and Hancock, P. J. B. (2009b). Looking at movies and cartoons: eye-tracking evidence from Williams syndrome and autism. J. Intellect. Disabil. Res. 53, 169-181.

Rutter, M., Bailey, A., and Lord, C. (2003). Social Communication
Questionnaire. Los Angeles, CA: Western Psychological Services.

Schultz, R. T., Grelotti, D. J., Klin, A., Kleinman, J., Van der Gaag, C., Marois, R., and Skudlarski, P. (2003). The role of the fusiform face area in social cogntion: implications for the pathobiology of autism. Philos. Trans. R. Soc. B Biol. Sci. 358, 415-427.

Singer Harris, N. G., Bellugi, U., Bates, E., Jones, W., and Rossen, M. (1997). Contrasting profiles of language development in children with Williams and Down syndrome. Dev. Neuropsychol. 13, 345-370.

Stojanovik, V. (2006). Social interaction deficits and conversational inadequacy in Williams syndrome. J. Neurolinguistics 19, 157-173.

Stojanovik, V., Perkins, M., and Howard, S. (2001). Language and conversational abilities in Williams syndrome: how good is good? Int. J. Lang. Commun. Disord. 36(Suppl.), 234-240.

Stone, C. A. (1998). The metaphor of scaffolding: its utility for the field of learning disabilities. J. Learn Disabil. 31, 344-364.

Tager-Flusberg, H., Boshart, J., and Baron-Cohen, S. (1998). Reading the windows to the soul: evidence of domain-specific sparing in Williams syndrome. J. Cogn. Neurosci. 10, 631-639.

Tager-Flusberg, H., Plesa-Skwerer, D., Faja, S., and Joseph, R. M. (2003). People with Williams syndrome process faces holistically. Cognition $89,11-24$.

Tager-Flusberg, H., and Sullivan, K. (2000). A componential view of theory of mind: evidence from Williams syndrome. Cognition 76, 59-89.

Tassabehji, M., Hammond, P., Karmiloff-Smith, A.,Thompson, P., Thorgeirsson, S. S., Durkin, M. E., Popescu, N. C., Hutton, T., Metcalfe, K., Rucka, A., Stewart, H., Read, A. P., Maconochie, M., and Donnai, D. (2005). GTF2IRD1 in craniofacial development of humans and mice. Science 310, 1184

Tomc, S. A., Williamson, N. K., and Pauli, R. M. (1990). Temperament in Williams syndrome. Am. J. Med. Genet. 36, 345-352.

Udwin, O. (1990). A survey of adults with Williams syndrome and idiopathic infantile hypercalcaemia. Dev. Med. Child Neurol. 32, 129-141.

Udwin, O., and Yule, W. (1991). A cognitive and behavioural phenotype in Williams syndrome. 
J. Clin. Exp. Neuropsychol. 13, Wood, D., Bruner, J. S., and Ross, G. 232-244.

Vygotsky, L. S. (1978). Mind and Society: The Development of Higher Psychological Processes. Cambridge, MA: Harvard University Press.

Wang, P. P., Doherty, S., Rourke, S. B., and Bellugi, U. (1995). Unique profile of visuo-perceptual skills in a genetic syndrome. Brain Cogn. 29, 54-65. (1976). The role of tutoring in problem solving. J. Child Psychiatry Psychol. 17, 89-100.

Conflict of Interest Statement: The conducted in the absence of any commercial or financial relationships that could be construed as a potential conflict of interest. authors declare that the research was
Received: 20 February 2012; paper pending published: 13 March 2012; accepted: 27 May 2012; published online: 25 June 2012.

Citation: van der Fluit F, Gaffrey MS and Klein-Tasman BP (2012) Social cognition in Williams syndrome: relations between performance on the social attribution task and cognitive and behavioral characteristics. Front. Psychology 3:197. doi: 10.3389/fpsyg.2012.00197
This article was submitted to Frontiers in Developmental Psychology, a specialty of Frontiers in Psychology.

Copyright (c) 2012 van der Fluit, Gaffrey and Klein-Tasman. This is an open-access article distributed under the terms of the Creative Commons Attribution Non Commercial License, which permits noncommercial use, distribution, and reproduction in other forums, provided the original authors and source are credited. 